\title{
Non-destructive evaluation of moisture content in wood using ground-penetrating radar
}

\author{
Hamza Reci ${ }^{1}$, Tien Chinh Maï ${ }^{2}$, Zoubir Mehdi Sbartaï ${ }^{2}$, Lara Pajewski ${ }^{3}$, and Emanuela Kiri ${ }^{1}$ \\ ${ }^{1}$ Institute of Geosciences, Energy, Water and Environment, Polytechnic University, Tirana, 1024, Albania \\ ${ }^{2}$ I2M Laboratory, Department of Environmental Civil Engineering, University of Bordeaux, 33405, Talence, France \\ ${ }^{3}$ Department of Information Engineering, Electronics and Telecommunications, Sapienza University of Rome, \\ 00184, Rome, Italy
}

Correspondence to: Hamza Reci (reci.jack@gmail.com)

Received: 1 July 2016 - Published in Geosci. Instrum. Method. Data Syst. Discuss.: 18 July 2016

Revised: 24 October 2016 - Accepted: 6 November 2016 - Published: 16 December 2016

\begin{abstract}
This paper presents the results of a series of laboratory measurements, carried out to study how the groundpenetrating radar (GPR) signal is affected by moisture variation in wood material. The effects of the wood fibre direction, with respect to the polarisation of the electromagnetic field, are investigated. The relative permittivity of wood and the amplitude of the electric field received by the radar are measured for different humidity levels using the direct-wave method in wide angle radar reflection configuration, in which one GPR antenna is moved while the other is kept in a fixed position. The received signal is recorded for different separations between the transmitting and receiving antennas. Dielectric constants estimated from direct waves are compared to those estimated from reflected waves: direct and reflected waves show different behaviour when the moisture content varies, due to their different propagation paths.
\end{abstract}

\section{Introduction}

Ground-penetrating radar (GPR) is an effective technique that uses electromagnetic waves to obtain three-dimensional images of natural or man-made structures and subsoil. It is employed in a large variety of applications for which noninvasive and non-destructive investigations are required. Examples of applications are surveying of transport infrastructures and buildings, detection and location of utilities, inspection of construction materials, geological and geotechnical investigations, archaeological prospecting and culturalheritage diagnostics, detection of landmines and unexploded ordnance, planetary exploration and more (Benedetto and $\mathrm{Pa}$ jewski, 2015; Persico, 2014).

Recently, GPR started being used for the non-destructive evaluation of moisture content in wood material. The most common causes of wood deterioration are biological, due to infestation of fungi and insects. There are several factors influencing the biological degradation of wood caused by fungi and insects in addition to moisture, for example, the source of infection, substrate (food), oxygen, temperature and more. However, the moisture content is recognised as one of the most critical factors for the development of such organisms. Fungal spores do not germinate readily on wood if the moisture content is below the fibre saturation point, commonly reached around $25-30 \%$. The percentage of moisture that is required for wood rotting fungi to flourish depends on the species of fungi and on the kind of wood. It is therefore apparent that the non-destructive evaluation of moisture content is of primary importance for the preservation of timber structures.

Few works in the literature are concerned with the GPR inspection of wood. In Lualdi et al. (2003), Muller (2003), Sbartaï (2011), Martínez-Sala et al. (2013b), Mai et al. (2015), the properties of wood were estimated using reflected-wave methods. In Lualdi et al. (2003), GPR was used to detect timber beams and evaluate the type and size of their connection to a bearing wall. In Muller (2003), GPR was employed for the inspection of timber bridges in order to detect piping and rotting defects. In Martínez-Sala et al. (2013b), GPR was used on samples of sawn timber of different species (densities) and interesting results were 
found: the propagation velocities, as well as the amplitudes of the reflected waves, were always smaller when the electric field was longitudinal to the grain rather than transverse to it. However, when the field propagated in a random direction, the electromagnetic parameters did not differ significantly. In Mai et al. (2015), laboratory measurements were carried out with the aim of studying the sensitivity of electromagneticwave propagation to moisture variation and fibre direction, in spruce and pine wood samples. The relative permittivity (dielectric constant) was measured using the resonance technique at $1.26 \mathrm{GHz}$, and results were compared to GPR measurements carried out with a $1.5 \mathrm{GHz}$ ground-coupled antenna. The samples were tested in different moisture-content conditions, ranging from 0 to $30 \%$ by mass. The real part of the permittivity estimated using the GPR technique turned out to be in good agreement with that measured by the resonance technique. The effect of moisture content when the electric field was oriented parallel to the fibre direction was observed to be more significant than when the electric field was oriented orthogonal to the fibres.

In Laurens et al. (2005), Sbartaï et al. (2006a, b), MartínezSala et al. (2013a), direct-wave methods were used for the non-destructive evaluation of concrete properties, with successful results. Direct-wave methods are of practical interest, because sometimes it may be difficult to detect the signal reflected by a sample when applying the technique on site.

A preliminary example of application of the direct-wave approach to wood assessment can be found in Mai et al. (2015). In that paper, tests were carried out on a sample of spruce with a humidity of $12 \%$. Measurements were performed with the electric field orthogonal to the wood fibres. The obtained results showed that the direct-wave signal is measurable. In addition, the permittivity values estimated by employing the direct-wave technique turned out to be lower than those estimated from the reflected waves.

Our work focuses on using the GPR direct wave to estimate the properties of wood. Results are compared with those obtained by employing a reflected-wave approach. Different moisture-content conditions are reproduced in the laboratory and analysed. The effects of the wood fibre direction, with respect to the polarisation of the electromagnetic field, are investigated. An interesting feature of the direct-wave method is that the operator simply has to measure the wave propagating between the transmitter and receiver, without needing a reflector at the bottom of the sample or information about the sample thickness.

\section{Methods}

The electromagnetic signal received by GPR in the presence of a wood sample is affected by many parameters, such as moisture content, wood density, temperature and direction of fibres (Sahin and Nürgul, 2004; Laurens et al., 2005; Kasal and Tannert, 2010). They influence the electromagnetic- field attenuation, phase shift and polarisation (Lundegren et al., 2006).

Let us consider a plane electromagnetic wave propagating through wood in the $z$ direction. The propagation can be assumed as governed by a factor $e^{-\gamma z}$, with complex propagation constant

$\gamma=j \omega \sqrt{\varepsilon_{\mathrm{r}} \varepsilon_{0} \mu_{\mathrm{r}} \mu_{0}}=a+j \beta$,

where $\omega$ is the angular frequency of the wave, $\varepsilon_{\mathrm{r}}=\varepsilon^{\prime}-j \varepsilon^{\prime \prime}$ is the relative permittivity of wood, $\varepsilon_{0}=8.854 \times 10^{-12} \mathrm{Fm}^{-1}$ is the permittivity of a vacuum, $\mu_{\mathrm{r}} \approx 1$ is the relative permeability of wood; moreover, as is well known, $\varepsilon_{0}=1 /\left(\mu_{0} c_{0}^{2}\right)$, where $\mu_{0}=4 \pi \times 10^{-7} \mathrm{Hm}^{-1}$ is the permeability of a vacuum and $c_{0}=299792458 \approx 3 \times 10^{8} \mathrm{~m} \mathrm{~s}^{-1}$ is the speed of light in a vacuum. The real part of $\gamma$ can be defined as an attenuation constant

$\alpha=\frac{\omega}{c_{0}}\left[\frac{\varepsilon^{\prime}}{2}\left(\sqrt{1+\tan ^{2} \delta}-1\right)\right]^{\frac{1}{2}}$,

and the imaginary part of $\gamma$ can be defined as a phase constant

$\beta=\frac{\omega}{c_{0}}\left[\frac{\varepsilon^{\prime}}{2}\left(\sqrt{1+\tan ^{2} \delta}+1\right)\right]^{\frac{1}{2}}$,

where $\tan \delta=\varepsilon^{\prime} / \varepsilon^{\prime \prime}$ is the loss tangent of wood.

As already mentioned, electromagnetic waves propagate in a vacuum at an approximate speed of $0.3 \mathrm{~m} \mathrm{~ns}^{-1}$. In wood, the electromagnetic velocity can be expressed as follows:

$v=\frac{\omega}{\beta} \approx \frac{c_{0}}{\sqrt{\varepsilon_{\mathrm{r}}}}$.

In this work, GPR radargrams are recorded by using two different techniques: direct and reflected waves are measured by the wide angle radar reflection (WARR) and fixed offset (FO) methods, respectively, as will be explained in the following. Ground-coupled antennas are employed with a central frequency of $1.5 \mathrm{GHz}$; the radar system is a GSSI SIR 3000. A wood sample of Picea (spruce) type is used, which is $600 \mathrm{~mm}$ long, $190 \mathrm{~mm}$ wide and $176 \mathrm{~mm}$ thick (see Fig. 1). Measurements are carried out in two directions: longitudinal (as in Fig. 1a), for which the electric field is polarised orthogonal to the wood fibres and transversal (as in Fig. 1b), for which the electric field is parallel to the fibres. In order to easily distinguish between reflected and direct waves, a metallic sheet is placed under the wood sample.

Measurements start at a humidity level of the wood sample equal to $12 \%$, known as the reference humidity (water content) used for wood characterisation. We measured the reference humidity to be $12 \%$, at the end of the experiments by putting the sample to the oven and finding the weight in anhydrous mode. The calculated density at zero humidity is used to correct the humidity values. Afterwards, the sample 
Table 1. Relative permittivity of wood for different levels of humidity by mass water, for direct- and reflected-wave approaches.

\begin{tabular}{|c|c|c|c|c|c|}
\hline \multicolumn{3}{|c|}{ Direct-wave (WARR) method } & \multicolumn{3}{|c|}{ Reflected-wave method } \\
\hline $\begin{array}{l}\text { Humidity } \\
(\%)\end{array}$ & $\begin{array}{l}\text { Relative permittivity } \\
\text { ( } E \perp \text { wood fibres) }\end{array}$ & $\begin{array}{l}\text { Relative permittivity } \\
\text { ( } E / / \text { wood fibres) }\end{array}$ & $\begin{array}{l}\text { Humidity } \\
(\%)\end{array}$ & $\begin{array}{l}\text { Relative permittivity } \\
\text { ( } E \perp \text { wood fibres })\end{array}$ & $\begin{array}{l}\text { Relative permittivity } \\
\text { ( } E / / \text { wood fibres) }\end{array}$ \\
\hline 12 & 1.56 & 1.67 & 12 & 2.24 & 2.35 \\
\hline 18.18 & 1.57 & 1.71 & 18.66 & 2.36 & 4.38 \\
\hline 23.87 & 1.71 & 2 & 25.46 & 2.92 & 6.32 \\
\hline 26.89 & 1.87 & 2.09 & 28.99 & 2.96 & 6.81 \\
\hline 30.15 & 2.01 & 2.36 & 33.01 & 3.18 & 7.56 \\
\hline 42.9 & 2.54 & 2.8 & 44.69 & 3.9 & 8.65 \\
\hline 51.6 & 2.91 & - & 54.09 & 4.54 & 10.69 \\
\hline 64.65 & 3.12 & - & 66.14 & 5.72 & 12.73 \\
\hline
\end{tabular}

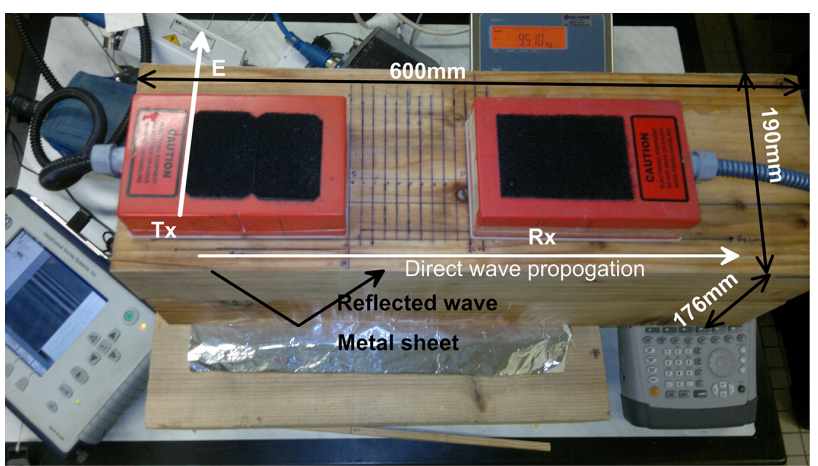

(a)

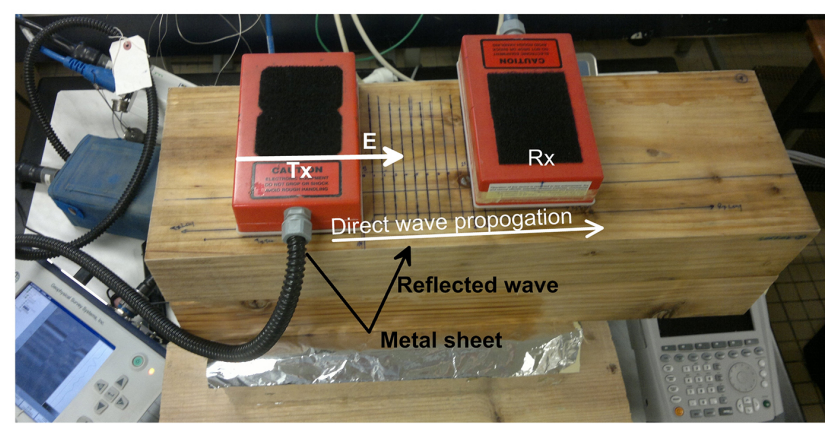

(b)

Figure 1. GPR measurements on the wood sample using groundcoupled antennas and the WARR technique. (a) Electric field is perpendicular to the fibres. (b) Electric field is parallel to the fibres.

is immersed in water to gradually increase its moisture content. GPR experiments are then repeated at different humidity levels.

Humidity by mass water (\%) is calculated by adopting the following expression (Moron et al., 2016):

Humidity $(\%)=\left(\frac{W-W_{0}}{W_{0}}\right) 100$,

where $W_{0}$, is the weight of the sample in anhydrous mode and $W$ is the weight of the sample after being immersed into the

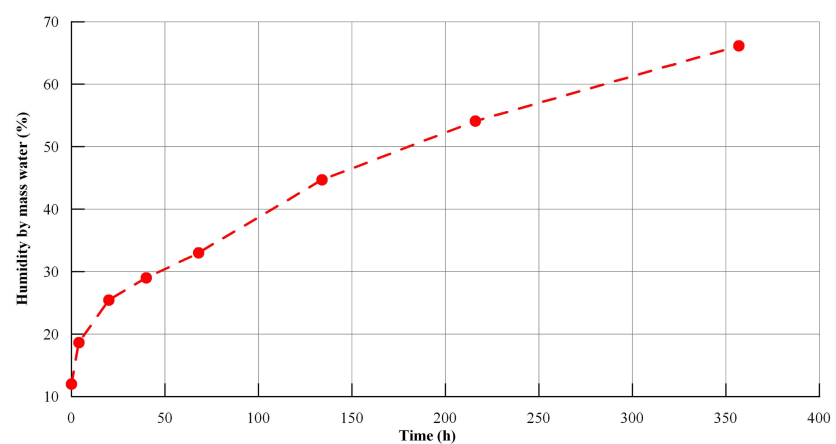

Figure 2. Humidity by mass water as a function of the time of immersion into the water.

water. The weight of the sample was measured with a balance which was sensitive to the gram. As already mentioned, the size of the sample is $600 \times 190 \times 176 \mathrm{~mm}$ and the density of the tested wood is about $450 \mathrm{~kg} \mathrm{~m}^{-3}$; therefore the sample at zero humidity has a mass of about $10 \mathrm{~kg}$ and the error due to balance precision is about $0.01 \%$.

Figure 2 shows the humidity by mass water of the sample, as a function of the time of immersion into the water. The measurements are performed at the humidity levels listed in Table 1.

When applying the WARR technique, a radar antenna is kept in a fixed position and the other antenna is moved on the wood surface with a $1 \mathrm{~cm}$ step. The distances between the two antennas vary from 16 to $26 \mathrm{~cm}$ and from 11 to $21 \mathrm{~cm}$ for orthogonal and parallel polarisations of the electric field, respectively. When the FO method is applied, the distances between the antennas are 16 and $11 \mathrm{~cm}$ for orthogonal and parallel polarisation states, respectively. The arrival times are visualised with Radan software and Matlab. Examples of Ascans and B-scans showing the superposition of direct-air wave, direct wave and reflected wave are provided in Figs. 3 and 4; here, the polarisation of the electric field is orthogonal to the fibres and the humidity level by mass water is $12 \%$. 


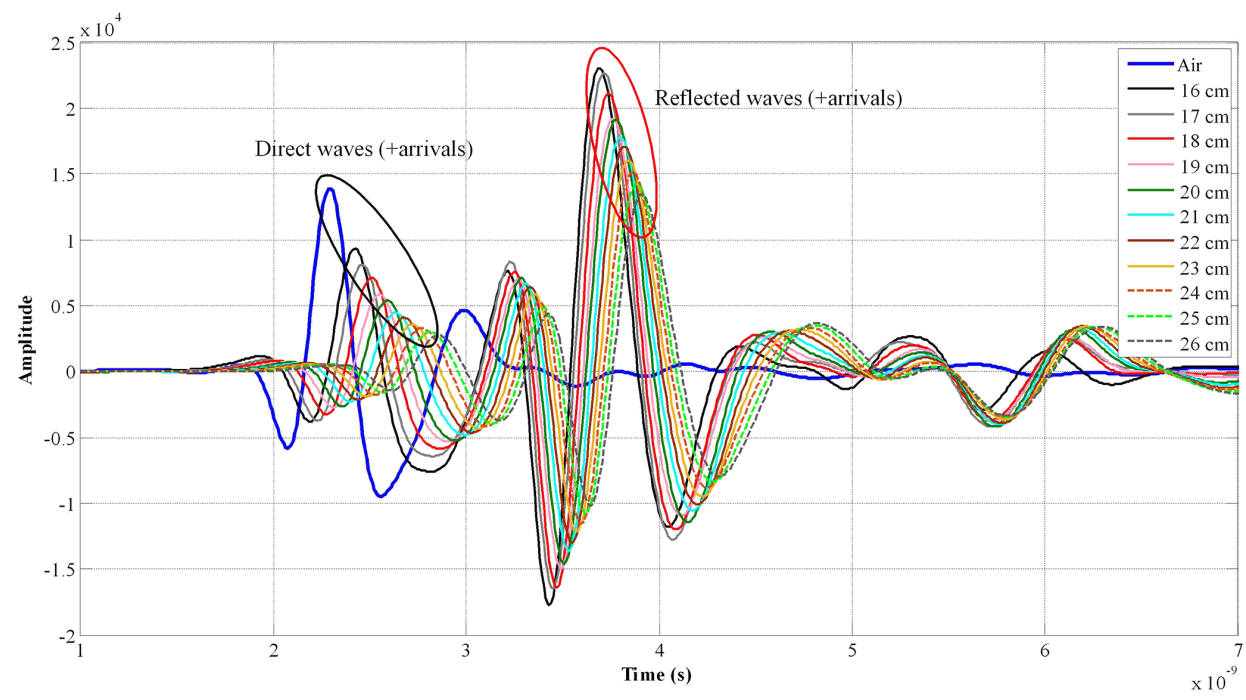

Figure 3. A-scans showing the superposition of the direct-air, direct and reflected waves measured over the sample when the polarisation of the electric field is orthogonal to the fibres and at a $12 \%$ humidity level by mass water.

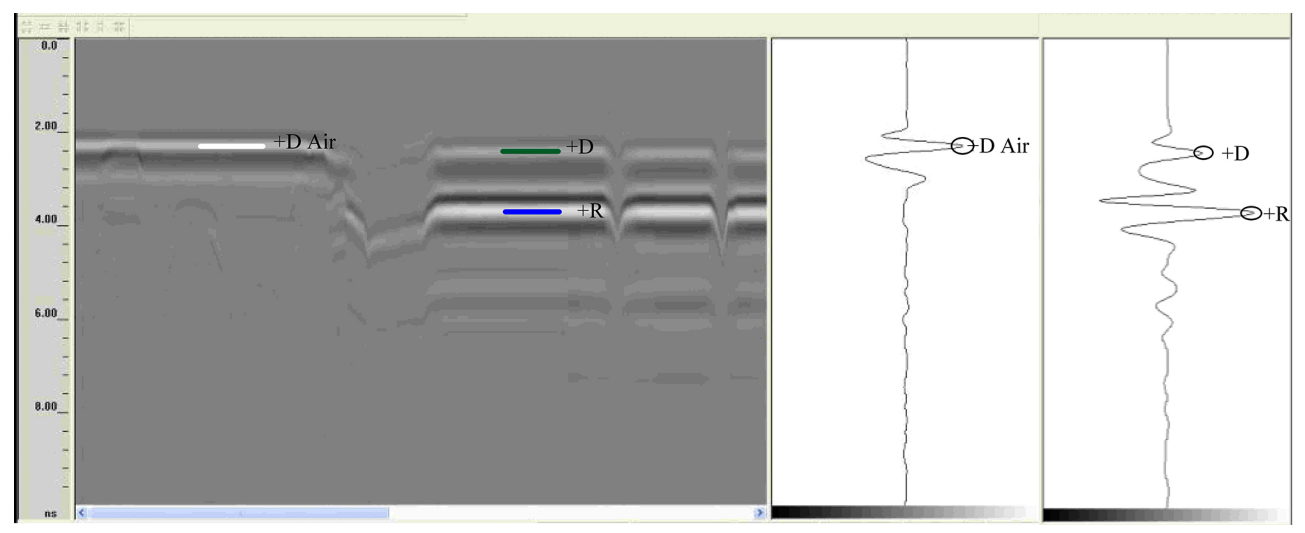

Figure 4. B-scans showing the direct-air, direct and reflected waves measured over the sample when the polarisation of the electric field was perpendicular to the fibres and at a $12 \%$ humidity level by mass water.

For the WARR technique, the propagation velocity is estimated from the arrival times of the direct waves, measured at difference distances between the antennas (the arrival time is the instant corresponding to the first and highest positive peak in the radargram). In particular, the propagation velocity is estimated as the slope of the linear regression of the arrival time of the direct wave, as a function of the distance between antennas. This is shown in Fig. 5, for both polarisation cases and a level of humidity equal to $18.18 \%$.

For the reflected wave, the propagation velocity $v$ in the wood sample was determined from the peaks of the air wave $(+D)$ and reflected wave $(+R)$ and the expression derived in the following was used (Fig. 4). For the direct air wave $(+D)$, the arrival time is

$t_{\mathrm{air}}=t_{0}+t_{\mathrm{air}}^{\mathrm{real}}=t_{0}+\frac{d_{\mathrm{TR}}}{c_{0}}$, where $t_{\text {air }}^{\text {real }}$ is the arrival time of the air wave (reference signal), $t_{0}$ is the starting time of electromagnetic impulse, $c_{0}$ as already mentioned - is the light velocity in a vacuum (as is well known, it is reasonable to assume that the velocity of light in the air on Earth is the same as in a vacuum), and $d_{\mathrm{TR}}$ is the distance between the transmitting and receiving antennas.

For the reflected wave $(+R)$ the arrival time is

$t_{\mathrm{r}}=t_{0}+t_{\mathrm{r}}^{\text {real }}=t_{0}+\frac{d_{\mathrm{R}}}{v}$,

where $t_{\mathrm{r}}^{\text {real }}$ is the arrival time of the reflected wave and $d_{\mathrm{R}}$ is the length of the propagation path of the reflected wave.

$d_{\mathrm{R}}=2 \sqrt{\left(\frac{d_{\mathrm{TR}}}{2}\right)^{2}+h^{2}}$,

where $h$ is the thickness of the wood sample (Fig. 6). 


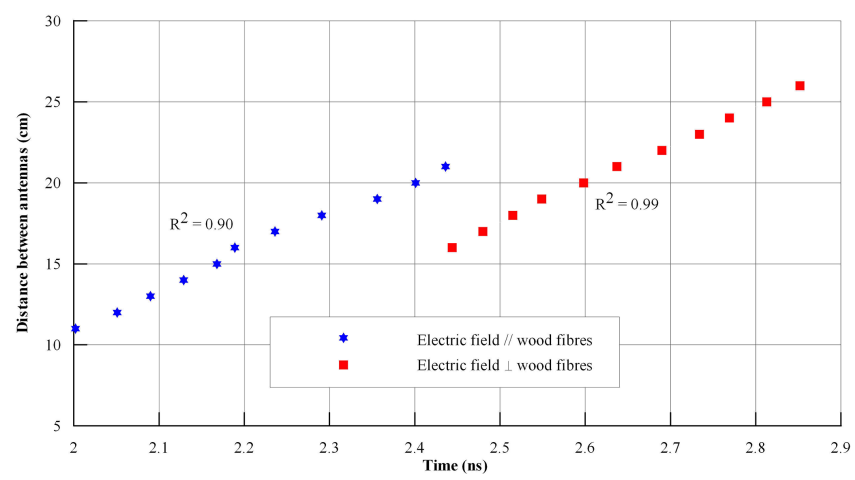

Figure 5. Determination of the propagation velocity for the direct wave from the arrival times. Both configurations with electric field parallel and perpendicular to the fibres are considered. In this case the humidity level was $18.18 \% . R^{2}$ is the coefficient for the determination of linear dependence.

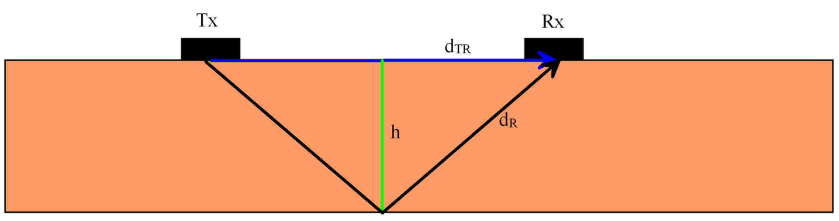

Figure 6. Schematic view of the distances between antennas (blue line), thickness of the wood sample (green line) and reflected-wave path (black line).

From the combination of Eqs. (7) and (8) it is possible to find the propagation velocity inside the wood sample, as follows:

$v=\frac{d_{\mathrm{R}}}{\Delta t+\frac{d_{\mathrm{TR}}}{c_{0}}}$,

where $\Delta t=t_{\mathrm{r}}^{\text {real }}-t_{\text {air }}^{\text {real }}$. Finally, the relative permittivity of the wood sample can be estimated from the following expression:

$\varepsilon_{\mathrm{r}} \approx\left(c_{0} / v\right)^{2}$

\section{Results and discussion}

As mentioned in Sect. 2, the wood relative permittivity is measured for different humidity levels (ranging from 12 to $64.5 \%$ ) and polarisation cases (electric field orthogonal and parallel to the wood fibres). Results are summarised in Table 1 and plotted in Fig. 7.

When the direct-wave method is used, the estimated value of the relative permittivity does not significantly change if the polarisation is rotated. When the electric field is parallel to the fibres, the permittivity values are systematically higher than those measured when the electric field is orthogonal to the fibres. Wood is an anisotropic media, so the dielectric

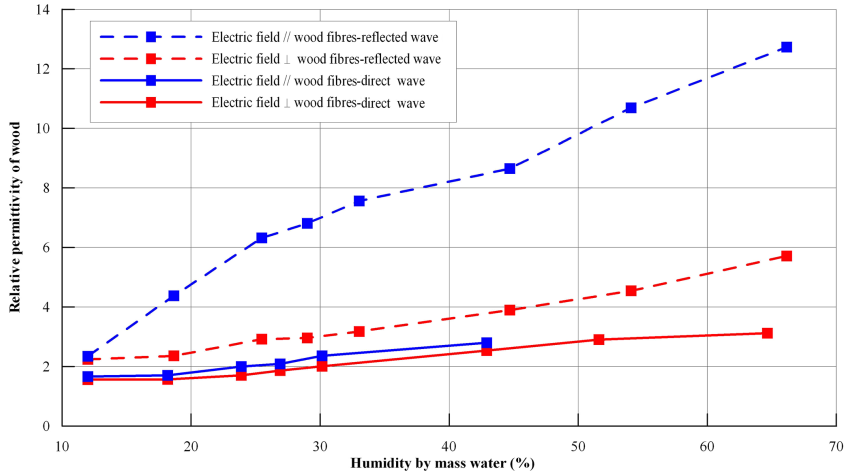

Figure 7. Relative permittivity as a function of humidity by mass water, estimated using the direct-wave (WARR) and reflected-wave methods for both polarisation cases.

properties of it are strongly influenced from the polarisation of electric field in relation to wood grains; moreover these properties are influenced by cellulose and mannan in the case of parallel polarisation, but in a transverse direction the dielectric properties are influenced by lignin. Lignin has lower dielectric properties than cellulose. Therefore, it is expected that the values of dielectric constants in the parallel polarisation are more influenced by humidity than in a transverse direction. In the case of the reflected wave, the electric field can be polarised exactly to the wood grains.

The increase of relative permittivity versus moisture content is piecewise linear, with a slope change occurring when the humidity level is about $18 \%$, in agreement with a previous publication where more samples were considered (Mai et al., 2015).

For the reflected-wave method, the increase of relative permittivity versus moisture content is piecewise linear as well, with a higher slope than in the case of the direct-wave method. Moreover, the slope strongly depends on the polarisation of the electromagnetic field and this is in agreement with Martínez-Sala et al. (2013b), Mai et al. (2015). When the electric field is orthogonal to the wood fibres, a slope change occurs at a humidity level of about $18 \%$, corresponding to the fibre saturation point. The slope change is less visible and seems to occur at higher humidity levels when the electric field is parallel to the wood fibres; this is again in agreement with Martínez-Sala et al. (2013b), Mai et al. (2015).

At all humidity levels, the permittivity values measured by the reflected-wave method are consistently higher than those measured by the direct-wave method. For both methods, the direction of the fibres does not affect the wood permittivity when the moisture content is low, then it becomes more important in the presence of higher humidity levels.

It is interesting to notice that the results of the reflectedwave method are closer to the direct-wave curves when the electric field is orthogonal to the wood fibres. When the electric field is polarised in a transverse direction, the dielectric 
properties of wood are influenced by lignin. Lignin has lower dielectric properties than cellulose and this could be the reason for this small change.

The obtained results show that direct waves in wood behave differently than reflected waves. This happens because the direct and reflected waves follow different propagation paths: the direct waves propagate in the top layer of the sample and the effect of the electromagnetic-field polarisation is small; the reflected waves propagate through the whole sample and, due to the anisotropy of wood, the polarisation has a stronger effect on the results.

When the electric field is orthogonal to the wood fibres, direct waves can be distinguished even when the humidity levels are above $60 \%$. When the electric field is parallel to the wood fibres, the direct-wave arrival time cannot be detected for humidity levels higher than $43 \%$. We tested humidity levels higher than $43 \%$, but in such conditions a high dissipation of electromagnetic energy occurs, the signal is completely attenuated in the parallel polarisation and it is not possible to extract signal parameters.

A further goal of this work is to study how the distance between the radar antennas affects the amplitude of the received signal. For each considered humidity level, the amplitude of the direct wave is then measured with antennas placed at 30 different distances. In Fig. 8, the direct-wave amplitude normalised to the amplitude of the direct-air wave is plotted as a function of the distance between transmitting and receiving antennas, when the humidity by mass water is $18.18 \%$. As expected, the amplitude shows an exponential attenuation when the distance increases. In Fig. 9, the normalised amplitude of the direct wave is plotted as a function of the humidity level for both parallel and orthogonal polarisation cases, when the distance between the antennas is 11 and $16 \mathrm{~cm}$, respectively. It can be noticed that, when the moisture content increases, the normalised amplitude at short distances turns out to be higher than one when the electric field is orthogonal to the wood fibres. This may be due to a superposition of direct and direct-air waves. In particular, in this configuration the distance $T-R$ is small and the dielectric constant is also small; hence the velocity is high and the propagation time is small, which allows for the superposition of direct and direct-air waves.

For small humidity levels, the normalised amplitude increases with moisture content, then when moisture content is further increased, the normalised amplitude starts to decrease (this happens at about 30 and $25 \%$ humidity by mass water for orthogonal and parallel polarisation, respectively). This phenomenon should be investigated more in depth by carrying out further measurements on different kinds of wood (with different densities) in order to have a clear picture of it.

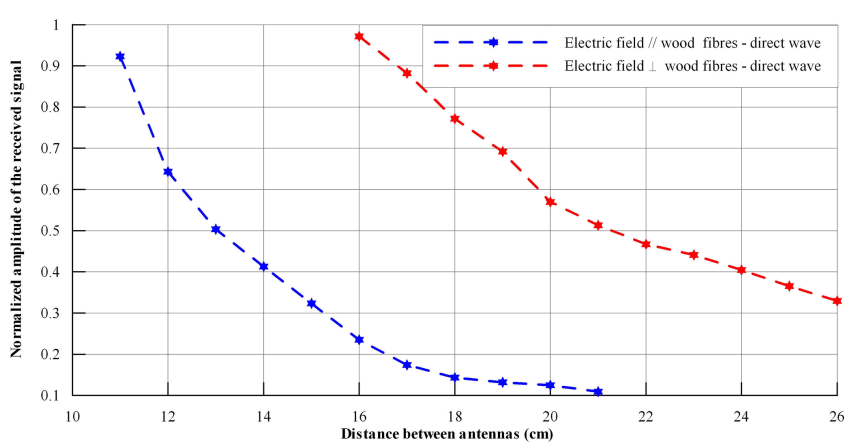

Figure 8. Amplitude of the signal received by the GPR, as a function of the distance between transmitting and receiving antennas (WARR method, $18.18 \%$ humidity by mass water).

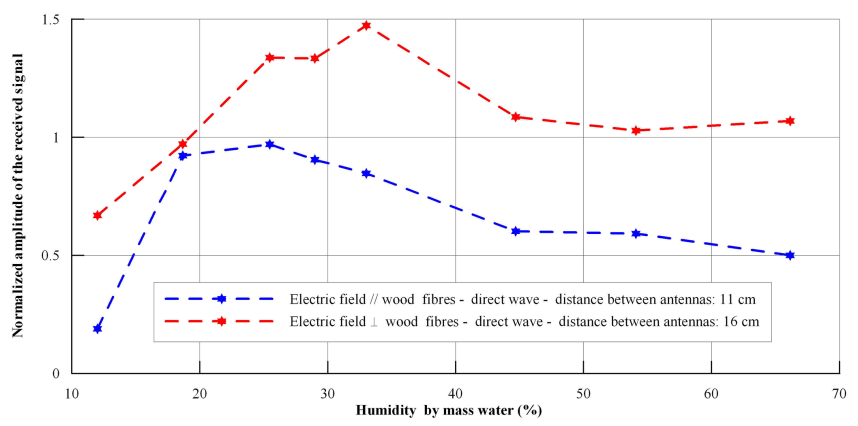

Figure 9. Normalised amplitude of direct wave with respect to humidity, for perpendicular and parallel polarisation of the electric field.

\section{Conclusions}

In this work, the sensitivity of ground-penetrating radar (GPR) signal to moisture variation in wood material was investigated. The relative permittivity of a Picea wood sample was measured at different humidity levels and for different polarisation states of the electromagnetic field.

Results obtained by using direct waves in wide angle radar reflection (WARR) configuration, in which one GPR antenna is moved while the other is in a fixed position, were compared to results obtained by using reflected waves in the so-called fixed offset configuration in which the distance between GPR antennas is fixed. Additionally, when the WARR method was applied, it was investigated how the attenuation of the received signal varies as a function of the distance between the radar antennas.

The presented results prove that direct and reflected waves have different behaviours when the moisture content varies, due to their different propagation paths. Overall, when the humidity levels increase, the difference between the permittivity values estimated by using the reflected- and directwave approaches becomes larger. 
For the reflected waves, the wood anisotropy affects the variation of the relative permittivity as a function of the moisture content; the effect is stronger when the electric field is parallel to the wood fibres. This is in good agreement with results available in the literature. Regarding direct waves, the measured values of the relative permittivity turn out to be weakly affected by the polarisation of the electromagnetic field. They are close to the values obtained by using reflected waves with the electric field orthogonal to the wood fibres. Apparently, the propagation paths are similar in the two cases.

Overall, our results show that the proposed measurement approach is effective at estimating the permittivity behaviour of wood material as a function of moisture content. The GPR technique is promising for moisture evaluation in timber structures and their early-stage diagnosis.

\section{Data availability}

GPR data in wood, relative to this paper are available in the online repository of IGEWE, http://www.geo.edu.al/ newweb/?fq=brenda\&gj=gj2\&kid=38.

Acknowledgements. The authors are grateful to COST (European Cooperation in Science and Technology, www.cost.eu) for funding the Action TU1208 "Civil Engineering Applications of Ground Penetrating Radar" (www.GPRadar.eu). The experimental results presented in this paper were collected at the University of Bordeaux, France, during a 1-month short-term scientific mission supported by TU1208. We acknowledge also the French National Research Agency (ANR) for supporting this study through the Xylo-plate project, Equipex XYLOFOREST. The authors are grateful to the EGU GI Division President, Francesco Soldovieri, the chief-executive editor of the Geoscientific Instrumentation, Methods and Data Systems journal, Jothiram Vivekanandan and the executive editors, Ari-Matti Harri and Håkan Svedhem, for inviting them to submit this paper, which resumes and extends the presentation (Reci et al., 2016) given during the GI3.1 Session of the 2016 EGU GA (Vienna, Austria, 17-22 April 2016).

Edited by: L. Eppelbaum

Reviewed by: two anonymous referees

\section{References}

Benedetto, A. and Pajewski, L. (Eds.): Civil engineering applications of Ground Penetrating Radar, in: Springer Transactions in Civil and Environmental Engineering, Springer International Publishing, doi:10.1007/978-3-319-04813-0, 2015.

Kasal, B. and Tannert, T. (Eds.): In situ assessment of structural timber: State of the art report of the RILEM Technical Committee 215-AS, in: RILEM state of the art reports, Springer, Heidelberg, New York, Series Volume 7, 2010.
Laurens, S., Balayssac, J. P., Rhazi, J., Klysz, G., and Arliguie, G.: Non-destructive evaluation of concrete moisture by GPR: experimental study and direct modelling, Mater. Struct., 38, 827-832, 2005.

Lualdi, M., Zanzi, L., and Binda, L.: Acquisition and processing requirements for high-quality $3 \mathrm{D}$ reconstructions from GPR investigations, International Symposium (NDT-CE 2003): NonDestructive Testing in Civil Engineering (NDT-CE) Berlin, 1619 September 2003.

Lundegren, N., Hagman, O., and Johansson, J.: Prediciting moisture content and density distribution of Scots pine by microwave scanning of sawn timber II: evaluation of models generated on a pixel level, J. Wood Sci., 52, 39-43, 2006.

Mai, T. C., Razafindratsima, S., Sbartaï, Z. M., Demontoux, D., and Frédéric Bos, F.: Non-destructive evaluation of moisture content of wood material at GPR frequency, Constr. Build. Mater., 77, 213-217, 2015.

Martínez-Sala, R., Rodríguez-Abad, I., and del Val, I.: Effect of penetration of water under pressure in hardened concrete on GPR signals, in: 7th International Workshop on Advanced Ground Penetrating Radar (IWAGPR), 2-5 July 2013, Nantes, France, doi:10.1109/IWAGPR.2013.6601523, 2013a.

Martínez-Sala, R., Rodríguez-Abad, I., Diez Barra R., and CapuzLladró, R.: Assessment of the dielectric anisotropy in timber using the nondestructive GPR technique, Constr. Build. Mater., 38 , 903-911, doi:10.1016/j.conbuildmat.2012.09.052, 2013b.

Moron, C., Garcia-Fuentevilla, L., Garcia, A., and Moron, A.: Measurement of moisture in wood for application in the restoration of old buildings, Sensors, 16, 607-705, 2016.

Muller, W.: Timber girder inspection using Ground Penetrating Radar, OR Insight, 45, 809-812, 2003.

Persico, R.: Introduction to Ground Penetrating Radar: Inverse Scattering and Data Processing, John Wiley \& Sons, Inc, Hoboken, NJ, 2014.

Reci, H., Sbart'i, Z. M., Pajewski, L., and Marciniak, M.: Moisture evaluation of wood material using GPR with WARR method COST Action TU1208, EGU General Assembly, Vienna Austria, 17-22 April 2016, EGU2016-18414-1, 2016.

Sahin, H. and Nürgul, A.: Dielectric properties of hardwood species at microwave frequencies, J. Wood Sci., 50, 375-380, 2004.

Sbartaï, Z. M.: Ground Penetrating Radar, in: In situ assessment of structural timber, edited by: Kasal, B. and Tannert, T., Springer Netherlands, 7, 25-37, doi:10.1007/978-94-007-0560-9_3, 2011.

Sbartaï, Z. M., Laurens, S., Balayssac, J. P., Ballivy, G., and Arliguie, G.: Effect of concrete moisture on radar signal amplitude, ACI Mater. J., 103, 419-426, 2006a.

Sbartaï, Z. M., Laurens, S., Balayssac, J. P., Arliguie, G., and Ballivy, G.: Ability of the direct wave of radar ground-coupled antenna for NDT of concrete structures, NDT\&E Int., 39, 400-407, 2006b. 